\title{
Edaravone promotes activation of resident cardiac stem cells by transplanted mesenchymal stem cells in a rat myocardial infarction model
}

\author{
Guang-Wei Zhang, MD, ${ }^{\mathrm{a}}$ Tian-Xiang Gu, MD, ${ }^{\mathrm{a}}$ Xue-Jun Sun, MD, ${ }^{\mathrm{b}, \mathrm{c}}$ Chunyue Wang, MD, ${ }^{\mathrm{d}}$ Xun Qi, MD, \\ Xiao-Bing Wang, $\mathrm{MD},{ }^{\mathrm{g}}$ and Jesse Li-Ling, $\mathrm{MD}, \mathrm{PhD}^{\mathrm{h}}$
}

\begin{abstract}
Objective: To explore the effect of edaravone on bone marrow mesenchymal stem cells (BMSCs) transplanted to treat acute myocardial infarction (AMI) and the underlying mechanism.

Methods: After pretreatment or treatment with edaravone under conditions of deep hypoxia and serum deprivation, the rat BMSCs were evaluated for reactive oxygen species (ROS), Akt pathway, apoptosis, migration, and paracrine function mediating cardiac stem cell (CSC) activation. Edaravone-pretreated BMSCs, control-released edaravone, and BMSCs were respectively transplanted into a rat AMI model. Apoptosis and paracrine functions of the BMSCs, resident CSC activation, and myocardial regeneration and function were measured at various time points.
\end{abstract}

Results: Compared with the control and edaravone pretreatment, edaravone treatment showed significantly increased apoptosis inhibition, migration, and cytokine secretion of BMSCs under an in vitro deep hypoxia and serum deprivation condition $(P<.05)$, via inhibiting intracellular accumulation of ROS and prolonging the Akt pathway activation. At 24 hours postoperatively, up-regulated expression of cytokines within the transplanted area, and decreased apoptotic BMSCs, were detected in the BMSC + edaravone group, compared with the BMSCs and edaravone pretreatment BMSC groups $(\mathrm{n}=10$ for each group, $P<.05)$. Four weeks later, the BMSCs + edaravone group showed more CSCs, CSC-derived cardiomyocytes, new vessels, and myocardial density within the ischemic area, and improved ejection fraction, compared with the other groups ( $\mathrm{n}=10$ in each group, $P<.05$ ).

Conclusions: Edaravone can protect the BMSCs against hypoxia and activate their potential to activate CSCs via the Akt pathway. The combined treatment can promote angiogenesis, resident CSC-mediated myocardial regeneration, and cardiac function after AMI, providing a new strategy for cell therapy. (J Thorac Cardiovasc Surg 2016;152:570-82)

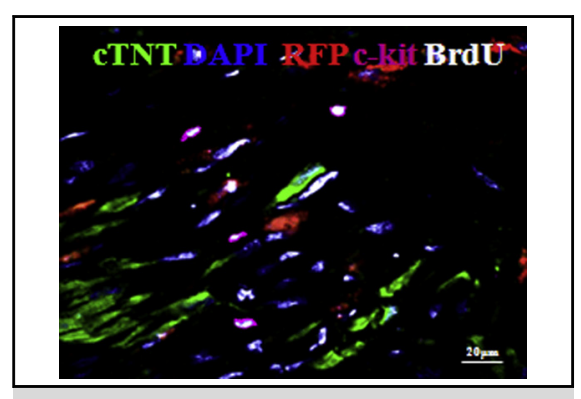

Edaravone and BMSC implantation promote CSC mediated myocardial regeneration.

\section{Central Message}

Edaravone can delay Akt pathway activation of BMSCs, and damage induced by hypoxia, to further promote angiogenesis and CSCmediated myocardial regeneration, and consequently ameliorate effects of BMSCs for AMI.

\section{Perspective}

Edaravone has been demonstrated to promote BMSC migration, paracrine and antiapoptosis, and enhance effects of BMSCs on CSC activation. A combination of edaravone and BMSC implantation increases myocardial regeneration after infarction, which may become a new strategy for cell therapies for acute ischemic myocardial injury, with wideranging clinical applications.

See Article page 345 .

See Editorial Commentaries page 350 and 583.
From the Departments of ${ }^{\mathrm{a}}$ Cardiac Surgery, ${ }^{\mathrm{b}}$ Anesthesiology, ${ }^{\mathrm{e}}$ Radiology, and ${ }^{\mathrm{g}}$ Echocardiography, and ${ }^{\mathrm{f}}$ Key Laboratory of Diagnostic Imaging and Interventional Radiology of Liaoning Province, The First Hospital of China Medical University, Shenyang; ${ }^{\mathrm{c} D e p a r t m e n t}$ of Anesthesiology of the First Affiliated Hospital of Dalian Medical University, Dalian; ${ }^{\mathrm{d}}$ Department of Cardiology, State Key Laboratory of Cardiovascular Disease, Cardiovascular Institute, Fuwai Hospital and National Center for Cardiovascular Diseases, Chinese Academy of Medical Sciences and Peking Union Medical College; ${ }^{\mathrm{h}}$ Institute of Genetic Medicine, School of Life Science, State Key Laboratory of Biotherapy, Sichuan University, Chengdu, China. This work was supported by The National Natural Science Foundation of China (No. 81400196), Science Technology Research Project of Education, Department of
Liaoning Province (No. L2011140, L2013297, and L2013316), and the Specialized Research Fund for the Doctoral Program of Higher Education (20132104110004). Received for publication Oct 11, 2015; revisions received Feb 23, 2016; accepted for publication Feb 28, 2016; available ahead of print April 5, 2016.

Address for reprints: Tian-Xiang Gu, MD, Department of Cardiac Surgery, The First Hospital of China Medical University, 155 Nanjingbei St, Shenyang 110001, China (E-mail: cmugtx@sina.com).

$0022-5223 / \$ 36.00$

Copyright (c) 2016 by The American Association for Thoracic Surgery http://dx.doi.org/10.1016/j.jtcvs.2016.02.071 

Abbreviations and Acronyms
$\mathrm{bFGF}=$ basic fibroblast growth factor
BMSC $=$ bone marrow mesenchymal stem cell
$\mathrm{BrdU}=5$-bromo-2-deoxyuridine
$\mathrm{CD}=$ cluster of differentiation
$\mathrm{CSC}=$ cardiac stem cell
cTnT $=$ cardiac troponin $\mathrm{T}$
CX43 = connexin 43
CXCR4 $=$ CXC chemokine receptor 4
HGF $=$ hepatocyte growth factor
IGF-1 = insulin-like growth factor 1
PCI = percutaneous coronary intervention
RFP = red fluorescent protein
ROS $=$ reactive oxygen species
VEGF $=$ vascular endothelial growth factor

\section{Scanning this QR code will take} you to the article title page.

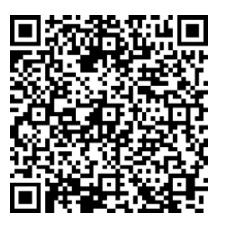

For its potential to improve cardiac function and reverse left ventricle remodeling, bone marrow mesenchymal stem cell (BMSC) transplantation has emerged as a promising strategy for the treatment of ischemic heart disease. ${ }^{1,2}$ However, BMSCs have to be transplanted into a severe hypoxic environment without any reperfusion in many patients because they cannot receive an incomplete revascularization even after percutaneous coronary intervention (PCI) or coronary artery bypass grafting treatment, owing to severe and complicated coronary artery disease. Therefore, their therapeutic effect has been restricted severely by the limited cell survival attributable to the hypoxic microenvironment of the transplant regions. ${ }^{3,4}$ Thus, protecting BMSCs against hypoxia may be critical for improvement of the efficiency of such therapy, ${ }^{5}$ especially when BMSCs are implanted directly into the core of ischemic regions during the surgery.

As a free radical scavenger, edaravone has been demonstrated to attenuate apoptosis of resident and engrafted cells within the ischemic regions, by inhibiting production of reactive oxygen species (ROS), which plays a critical role in hypoxia-induced injury. ${ }^{6,7}$ Although edaravone has been used in both animal experiments and clinical settings for the treatment of myocardial ischemia, ${ }^{8,9}$ as well as BMSC transplantation therapy for cerebral ischemia, ${ }^{10}$ whether this agent enhances the survival of BMSCs that are transplanted into ischemic cardiac regions is unclear.
To explore the effect of edaravone on BMSCs that are transplanted for the treatment of acute myocardial infarction and the underlying mechanism, we have designed an in vitro experiment to evaluate the potential of BMSCs against hypoxia, by measuring the following indices: ROS production, the Akt signal pathway, apoptosis, migration, paracrine function, and the effect of these on the CSCs. In addition, in vivo animal experiments were designed to assess the apoptosis and paracrine function of the transplanted BMSCs, as well as angiogenesis, resident CSC-mediated myocardial regeneration, and left ventricle function with the combined therapy, using controlled release of edaravone, and BMSC transplantation.

\section{METHODS}

All experiment procedures were approved by the Liaoning Administrative Committee for Laboratory Animals and were performed in strict compliance with "Guidance for the Care and Use of Laboratory Animals" from the National Institutes of Health.

\section{Preparation of BMSCs and CSCs in Vitro}

The BMSCs were isolated from the bone marrow of Sprague-Dawley rat extremities, with Ficoll density gradient centrifugation, ${ }^{11}$ and the CSCs were isolated from the left ventricle through collagenase digestion and immunomagnetic bead enrichment for $\mathrm{c}-\mathrm{kit}^{+} / \mathrm{CD} 45^{-}$cells. ${ }^{12,13}$ To avoid interference, the medium did not contain additional growth factors. As detected by fluorescence-activated cell sorting analysis, the BMSCs have expressed cluster of differentiation (CD)29 (91.32\%) and CD90 (92.75\%), but not CD34 (1.4\%) and CD45 (2.1\%), whereas the CSCs have expressed GATA-4 (57.89\%), Flk-1 (46.71\%), and Nkx2.5 $(35.93 \%)$, but not c-TNT $(0.17 \%)$ or CX-43 $(0.19 \%)$. The BMSCs were lentivirally transfected to express red fluorescent protein (RFP) as the label for transplantation.

Based on the knowledge that the local concentration of oxygen can be as low as $0.2 \%$ to $1 \%$ in the ischemic heart, deep hypoxia $(0.2 \%$ $\mathrm{O}_{2}$ ) and serum deprivation were used to imitate the in vivo microenvironment of the transplanted region by using a sealed GENbox hypoxic chamber. ${ }^{3}$ In keeping with the edaravone range of 10 to $1000 \mu \mathrm{M}$ found in a previous report, ${ }^{10} 500 \mu \mathrm{M}$ of edaravone (Nanjing Simcere Dongyuan Pharmaceutical, Nanjing, China) was used in the present in vitro experiments. The BMSCs $\left(4 \times 10^{6}\right)$ were exposed to this deep hypoxia/serum deprivation condition with or without edaravone pretreatment or treatment, to analyze the intracellular ROS and Akt pathway at 30 minutes, 2 hours, and 6 hours, respectively, cell migration at 8 hours, and apoptosis, cytokine secretion, and effects on the CSCs at 24 hours.

The CSCs were incubated with or without supernatant from the edaravone pretreated or treated BMSCs for the analysis of cell migration at 6 hours, apoptosis induced by deep hypoxia/serum deprivation at 8 hours, and differentiation into cardiomyocytes at 7 days, with single exposure to edaravone as the control, based on edaravone remaining in the supernatant from edaravone-treated BMSCs.

\section{Transplantation of BMSCs}

The rat model for acute myocardial infarction was constructed by ligating the left anterior descending artery. Thereafter, the animals were randomly assigned to 5 groups as shown in Figure $1(n=10$ in the control and edaravone groups; $\mathrm{n}=20$ in BMSCs-the edaravone pretreatment BMSCs and the BMSCs + edaravone groups). A total of $200 \mu \mathrm{l}$ of saline, with or without allogeneic BMSCs $\left(1 \times 10^{7}\right)$ and/or 
Control group $(\mathbf{n}=10)$

BMSCs group $(\mathrm{n}=\mathbf{2 0})$

ED group $(n=10)$

BMscs group $(\mathbf{n}=\mathbf{2 0})$

\section{A}

BMSCs+ED group $(\mathbf{n}=\mathbf{2 0}$

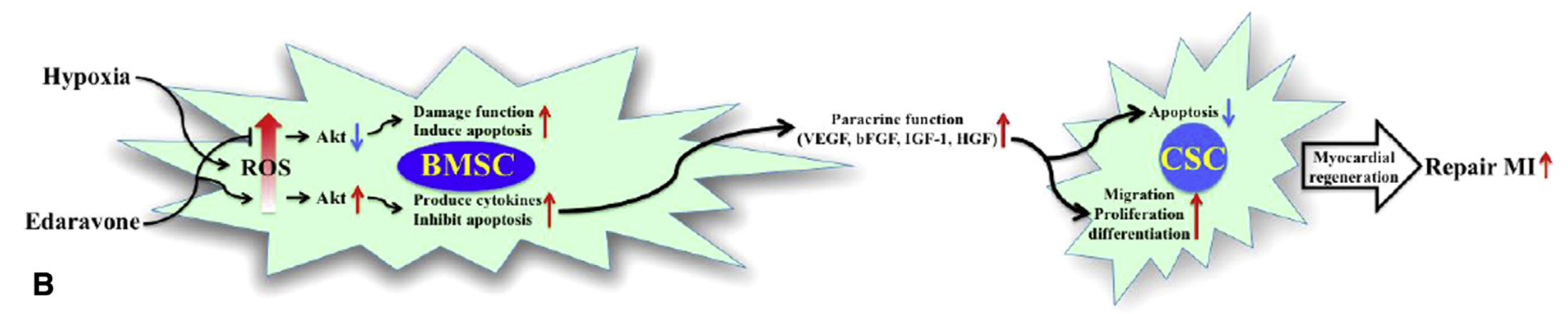

FIGURE 1. A, Flowchart of treatment in all groups: 24 hours after treatment, 10 samples from 10 animals were used for all analysis in the BMSC implantation groups. Four weeks later, 10 samples from 10 animals were used for all analysis in all groups. B, Flowchart of molecular pathway. BMSCs, Bone marrow mesenchymal stem cells; $E D$, edaravone; $R O S$, reactive oxygen species; $V E G F$, vascular endothelial growth factor; $b F G F$, basic fibroblast growth factor; $I G F-1$, insulin-like growth factor-1; $H G F$, hepatic growth factor; $C S C$, cardiac stem cell; $M I$, myocardial infarction.

edaravone $(10 \mathrm{mg} / \mathrm{kg})$ microspheres, were injected directly into the myocardium of the ischemic region at 2 sites. The microspheres (with a diameter ranging from 10 to $30 \mu \mathrm{m}$, and a release period of 2 to 3 weeks) were made, as described in our previous paper, ${ }^{14}$ from a biodegradable polymer material (poly D, L-lactic/glycolic acid, with no effect on activation of $\mathrm{BMSCs}^{15}$ ), which can fulfill the loading and controlled release of edaravone. After the operation, $50 \mathrm{mg} / \mathrm{kg} \mathrm{5-}$ bromo-2-deoxyuridine (BrdU) was administrated intraperitoneally twice a week to label the cells that were undergoing deoxyribonucleic acid replication. The ejection function was measured by echocardiography, to analyze cardiac function.

\section{Measurement of ROS Production and Apoptosis}

Intracellular ROS of the BMSCs was determined with a method described by Zhang and colleagues. ${ }^{16}$ Before the exposure to deep hypoxia/serum deprivation, the BMSCs were incubated for 30 minutes, with $20 \mu \mathrm{M}$ of $2^{\prime}, 7^{\prime}$-dichlorodihydrofluorescein diacetate (Sigma-Aldrich, St. Louis, Mo), which can be oxidized to dihydrofluorescein (green fluorescence) by ROS. Apoptosis of BMSCs and CSCs was evaluated with a flow cytometer after annexin V/propidium iodide staining.

\section{Enzyme-Linked Immunosorbent Assay, RT-PCR, and Western Blotting Analysis}

Enzyme-linked immunosorbent assay (R\&D Systems) was used to measure vascular endothelial growth factor (VEGF), basic fibroblast growth factor (bFGF), hepatocyte growth factor (HGF), and insulin-like growth factor 1 (IGF-1) secreted by the BMSCs into the supernatant. The messenger ribonucleic acid and protein levels of p-AKT/AKT, CXCR4, and caspase- 3 from the BMSCs, VEGF, bFGF, HGF, and IGF-1 from the BMSCs and tissue sample, and cTNT, $\alpha$-actinin, and Nkx2.5 from the CSCs, were respectively determined using real-time polymerase chain reaction and Western blotting with $\beta$-actin as the control.

\section{Analysis of Cell Migration}

An 8- $\mu \mathrm{m}$ pore-size Transwell migration chamber (Millipore, Billerica, Mass) was used for analyzing the migration of the BMSCs and CSCs. The cells were added to the upper chamber, and the stimuli were added to the lower chamber. Cells remaining on the upper face of the chambers were removed carefully with a cotton swab and subjected to crystal violet staining. Quantitative analysis was performed by measurement of the absorbance. 

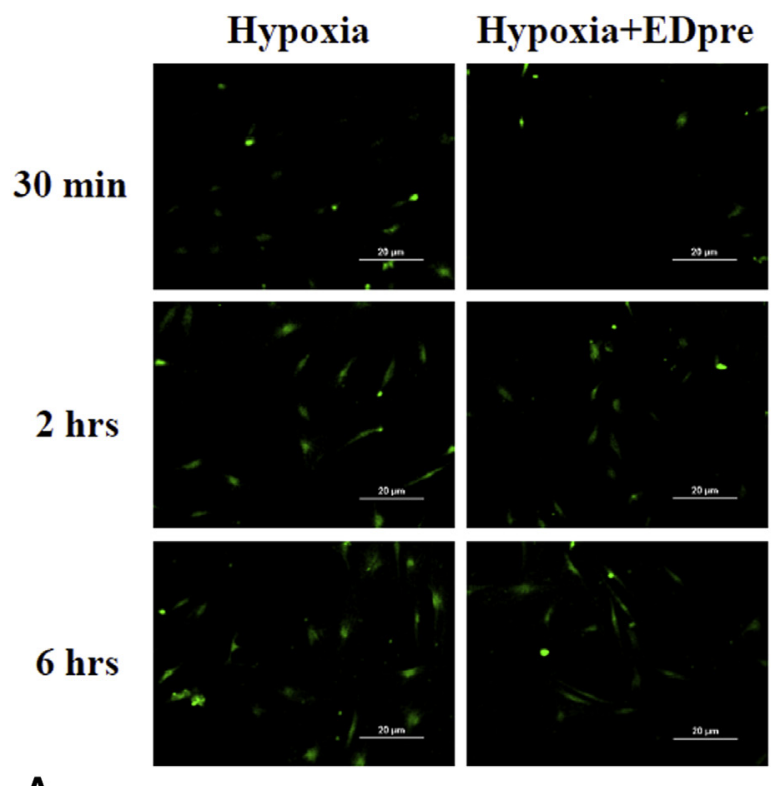

A

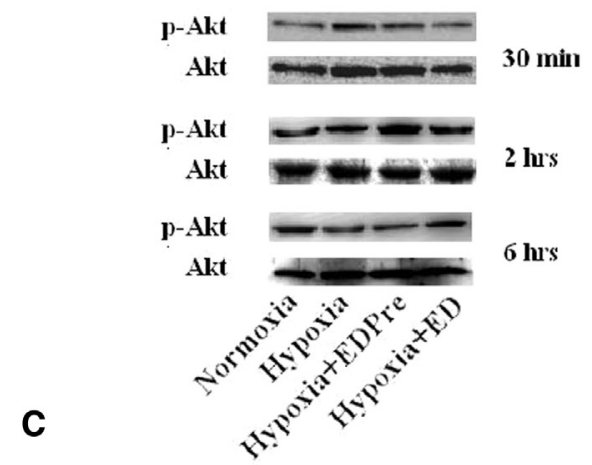

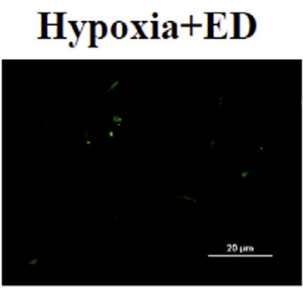
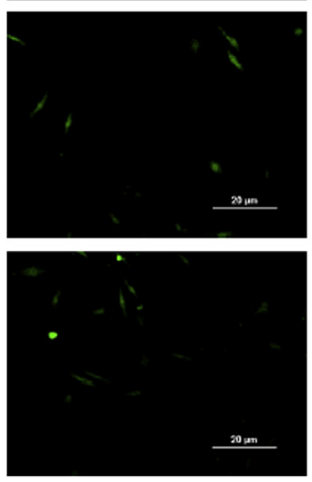

\section{Normoxia reference}
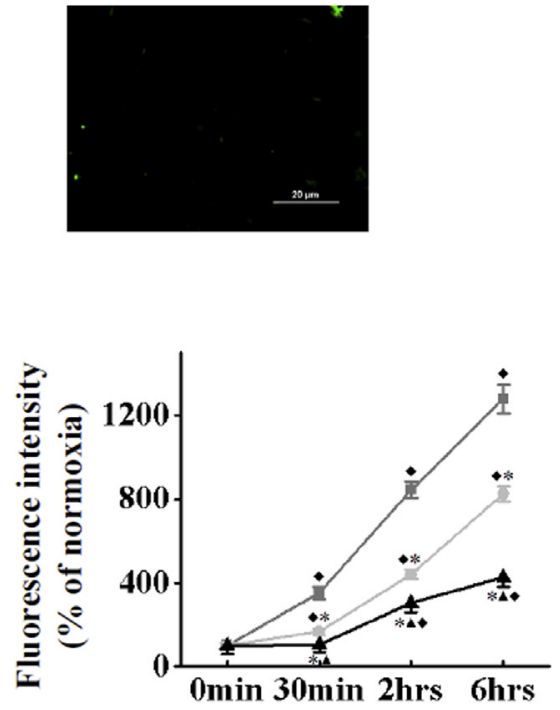

B

FIGURE 2. Ex vivo analysis of ROS production and Akt pathway of the BMSCs. A, Representative photos of DCF in BMSCs after 30 min, 2 hours, or 6 hours exposure to hypoxia, hypoxia + EDpre or hypoxia + ED (based on the fact that these images were from the same area of cell culture, and that the density of the cells is similar over time, the results at different time points are comparable to those without DAPI staining as the control). B, Changes of intracellular ROS production under each condition; C, representative results of Western blotting for p-Akt and Akt; D, Comparison of changes in p-Akt/Akt under various conditions. EDpre, Edaravone pretreatment. ${ }^{*} P<.001$ versus hypoxia. ${ }^{\star} P<.001$ versus hypoxia + EDpre. ${ }^{\star} P<.001$ versus normoxia (0 min).

\section{Histologic Analysis}

To confirm the differentiation of the CSCs, immunofluorescence of the lamella of the crawling cells was determined with anti-cTNT and anti-connexin43 (CX43) antibodies. Immunofluorescence of tissue sections was determined with anti-cTNT, anti-c-kit, anti-GATA4, and anti-BrdU antibodies (Abcam Ltd, Cambridge, United Kingdom) to identify the CSCs and nascent cardiomyocytes. Anti-von Willebrand factor staining was used to assess angiogenesis. Masson trichrome staining was performed to delineate the myofilament structure. Terminal deoxynucleotidyl transferase-mediated dUTP nick-end labeling staining was used to determine apoptosis of the transplanted BMSCs. For each animal or each sample, 5 nonoverlapping fields in sections were randomly photographed under a light or confocal microscope. Myocardial density and new vessels were quantified through optical density calibration.

\section{Statistical Analysis}

All data were measured and analyzed with a blind method. The results were presented as mean \pm standard deviation. A 1-way and a 2-way analysis of variance with least-squares post hoc correction were used to compare the measurements in each group using an SPSS 19.0 software package (SPSS Inc, Chicago, Ill).

\section{RESULTS}

The green fluorescence within the BMSCs has increased after 30 minutes of exposure to hypoxia and hypoxia + pretreatment edaravone, and after 2 hours of exposure to hypoxia + edaravone (Figure $2, A$ ). Semiquantitative analysis (Figure 2, B) suggested that the hypoxic condition caused the BMSCs to produce more ROS, compared with a normoxia condition $(P<.001)$, which continually increased with elapse of exposure time $(P<.001)$. Although the edaravone pretreatment and treatment both delayed such a process $(P<.05)$, the latter had a more significant effect $(P<.001)$. Western blotting 
assays (Figure 2, $C$ and $D$ ) revealed that the expression of phosphorylated-Akt was upregulated 30 minutes after the exposure to hypoxia $(P<.001)$, which fell to a nearly normal value at 2 hours $(P<.001)$, and became significantly downregulated at 6 hours $(P<.001)$. The edaravone pretreatment significantly delayed the upregulation and the fall of phosphorylated-Akt induced by hypoxia, and increased the peak value at 2 hours $(P<.001)$, but it did not inhibit the down-regulation at 6 hours $(P<.001)$, although the level was increased, compared with sole hypoxia $(P<.001)$. Only a mild up-regulation of phosphorylated-Akt occurred, $30 \mathrm{mi}$ nutes after the edaravone treatment and exposure to hypoxia $(P=.045)$, and it significantly increased at 2 hours and 6 hours $(P<.001)$, suggesting that edavarone prolonged the hypoxia-induced activation of the Akt pathway of the BMSCs.

\section{Edaravone Inhibited BMCS Apoptosis and Enhanced Their Migration and Paracrine Function Under Hypoxia, via the Akt Pathway}

As revealed by the Transwell migration assay (Figure 3,A), compared with exposure to normoxia, hypoxia significantly suppressed the migration of the BMSCs $(P<.001)$, which was partially inhibited by the edavarone treatment $(P<.001)$ but not by edaravone pretreatment (Figure 3, $B$; $P=1$ ). The annexin V/propidium iodide staining (Figure 3, $A$ ) showed that edaravone treatment significantly attenuated BMSC apoptosis, compared with exposure to hypoxia $(P<.001)$ and hypoxia + edaravone pretreatment $(P<.001)$. Meanwhile, no difference was found between the hypoxia and hypoxia + edaravone pretreatment groups $(P=.933)$. Up-regulated CXCR4 (a crucial molecule that enhances migration and adhesion of BMSCs under hypoxic conditions ${ }^{17}$ ) and down-regulated caspase-3 (a key downstream molecule that executes the apoptotic cascade after hypoxia $\left.{ }^{18}\right)$ expression was found after the edaravone treatment $(P<.001)$, compared with exposure to hypoxia and hypoxia + edaravone pretreatment.

To assess the paracrine function of the BMSCs induced by hypoxia and edaravone pretreatment or treatment, the messenger ribonucleic acid and protein expression (Figures 3, $D$ and $E$ ) and secretion of VEGF, bFGF, HGF, and IGF-1 were determined 24 hours after the treatment. Both real-time polymerase chain reaction and Western blotting analysis (Figure 3, $F$ and $G$ ) have revealed an increase in the intracellular expression of VEGF, bFGF, HGF, and IGF-1 under a hypoxic condition $(P<.05)$. The edaravone pretreatment could promote the increase induced by hypoxia $(P<.05)$, and a further increase was detected with the edaravone treatment $(P<.05)$. The ELISA assay has detected similar changes in the secretion of the 4 cytokines (Figure 3, I; $P<.05$ ).

LY294002, a PI3K inhibitor upstream of Akt, significantly attenuated the effect of edaravone treatment on
BMSC migration, apoptosis, and paracrine function under a hypoxic condition $(P<.05)$, indicating that the Akt pathway is involved in the protective mechanism of edaravone against hypoxia.

\section{In Vitro Apoptosis, Migration, and Differentiation of CSCs Induced by the BMSCs}

To assess the effect of edaravone pretreated and treated BMSCs, the apoptosis, migration, and differentiation of the CSCs were studied in vitro. The CSC migration was significantly promoted by a supernatant derived from normoxia-cultured BMSCs, compared with the control $(P<.001$; Figure 4, $A$ and $B)$. Hypoxia-culture enhanced the effect of BMSCs on CSC migration $(P<.001)$, which was further enhanced by the edaravone pretreatment $(P<.001)$. Similarly, supernatant derived from edaravone-incubated BMSCs under a hypoxic condition exerted the most significant effect on the CSC migration $(P<.001)$. Similar changes were detected with apoptosis (Figure $4, A$ and $C$ ) and expression of cTNT, $\alpha$-actinin, and $\mathrm{Nkx} 2.5$ (Figure $4, D$ and $E$ ), to identify CSC differentiation into cardiomyocytes $(P<.05)$, which was further supported by anti-cTNT and anti-CX43 immunofluorescence staining (Figure 4, F).

\section{In Vivo Apoptosis and Cytokine Expression of the Transplanted BMSCs}

At 24 hours after the transplantation, terminal deoxynucleotidyl transferase-mediated dUTP nick-end labeling staining detected a large number of apoptotic BMSCs that had been transplanted into the ischemic regions $(89.7 \% \pm 3.9 \%$; Figure $5, A)$. The ratio was lower, at $71.3 \% \pm 4.6 \%$ in the BMSCs + edaravone group $(P<.001)$, but it did not change in the edaravone pretreatment BMSC group $(87.7 \% \pm 3.8 \%, P=.871$; Figure $5, D)$. Expression of VEGF, bFGF, HGF, and IGF-1 was assessed by real-time polymerase chain reaction (Figure $5, B$ ) and Western blotting analysis (Figure 5,C). Compared with the BMSC group, the expression levels of such cytokines were significantly up-regulated in the edaravone pretreatment BMSC group (except IGF-1; $P<.001$ ), and a further increase was detected in the BMSCs + edaravone group $(P<.001)$, suggesting that the paracrine function of BMSCs was enhanced by control-released edaravone as well.

\section{Identification of Resident CSCs and Regenerated Cardiomyocytes After BMSC Transplantation}

At 4 weeks after the treatment, frozen tissue sections showed $\mathrm{RFP}^{+}$cells in the core of the ischemic region (the center of ischemic regions in which ischemia is the most severe) of the BMSC transplantation groups. The $\mathrm{RFP}^{-}$cells were confirmed as the resident. Photos of immunofluorescence staining are shown in Figure 6, A. Among the 5 groups, the

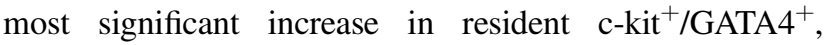



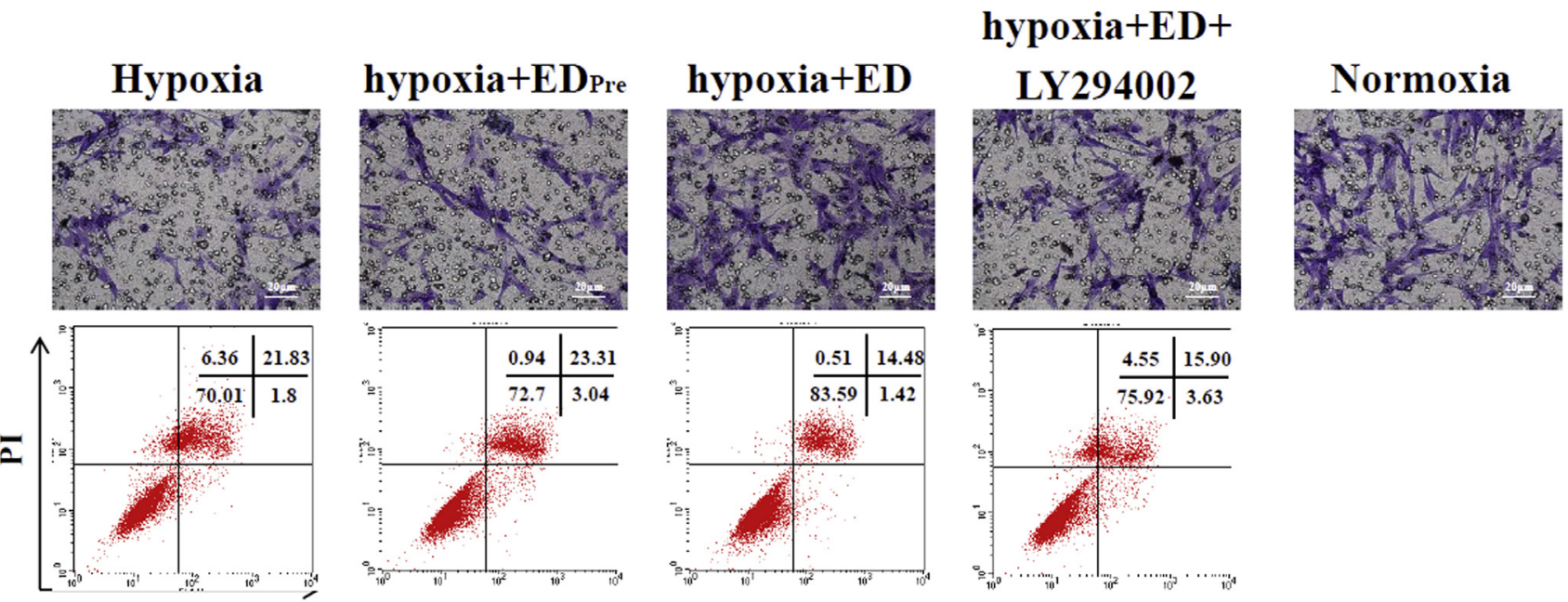

Annexin V-FITC
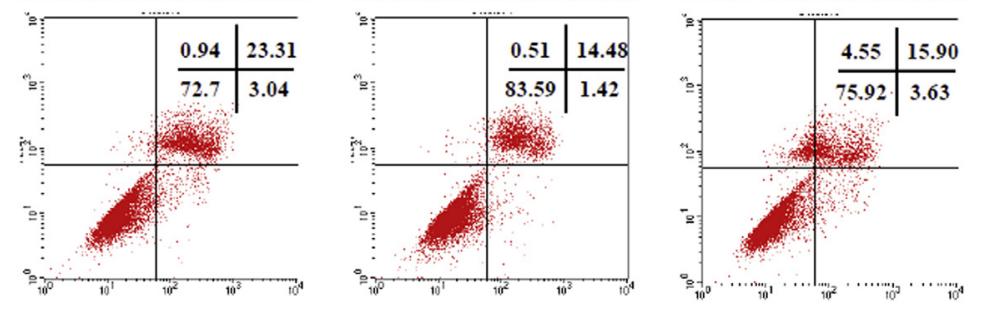

A

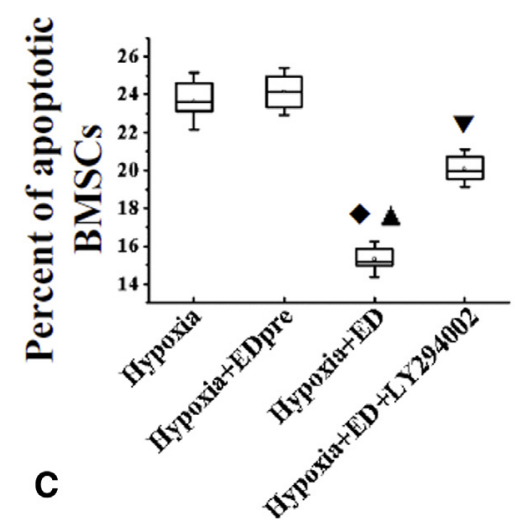

B

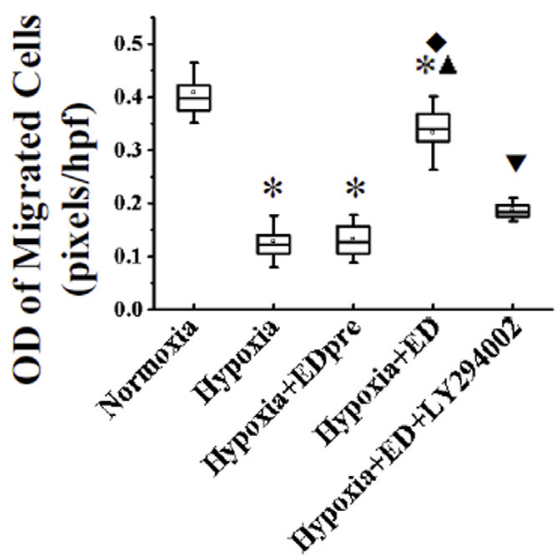

FIGURE 3. Ex vivo assessment of BMCS apoptosis, migration, and paracrine function. A, Representative photographs of (A) BMSC migration and (B, C) FACS images of Annexin V/PI staining for the assessment of BMSC apoptosis. The crystal violet optical density values of migrated BMSCs and the ratios of apoptotic cells are compared in (B) and (C), respectively. Representative photographs of electrophoresis of real time polymerase chain reaction and Western blotting products are respectively shown in (D) and (E) for the determination of the expression of CXCR4, Caspase 3, VEGF, bFGF, HGF, and IGF-1 in BMSCs after 24 hours of exposure, and quantitative comparison was performed in $(\mathrm{F}),(\mathrm{G})$, and $(\mathrm{H})$. I, Comparison of secreted cytokines measured by ELISA. EDpre, Edaravone pretreatment; $O D$, optical density; $B M S C$, bone marrow mesenchymal stem cell; VEGF, vascular endothelial growth factor; $b F G F$, basic fibroblast growth factor; $H G F$, hepatic growth factor; IGF-1, insulin-like growth factor-1; CXCR4, chemokine (C-X-C motif) receptor 4 . ${ }^{*} P<.001$ versus normoxia. ${ }^{\wedge} P<.001$ versus hypoxia. ${ }^{\star} P<.001$ versus hypoxia + EDpre. ${ }^{\nabla} P<.001$ versus hypoxia + ED.

$\mathrm{BrdU}^{+} / \mathrm{c}-\mathrm{kit}^{+}$and $\mathrm{BrdU}^{+} / \mathrm{cTNT}^{+}$cells was found in the BMSCs + edaravone group $(P<.001)$, although an increase occurred in the edaravone pretreatment BMSC group as well $(P<.001)$, compared with the other 3 groups. In addition, more such cells were found in the edaravone group $(P<.001)$ and the BMSC group $(P<.001)$, compared with the control group (Figure $6, B$ ).

\section{Analysis of Angiogenesis, Myocardium Density, and Cardiac Function}

For analysis of angiogenesis, anti-von Willebrand factor staining (Figure 7, A) revealed that new vessel density was significantly increased in BMSCs, edaravone, edaravone pretreatment BMSCs, and BMSCs + edaravone groups, compared with the control $(P<.001$; Figure 7 , $B)$. A significant increase occurred in the edaravone pretreatment BMSC group $(P<.001)$, and a further increase in the BMSCs + edaravone group $(P<.001)$, compared with the BMSCs and edaravone groups. Similar to the anti-von Willebrand factor staining results, the most viable myocardium was in the BMSCs + edaravone group $(P<.001$; Figure 7, $C)$.

At 4 weeks after the treatment, a significant improvement had occurred in ejection fraction in the BMSC group, the 


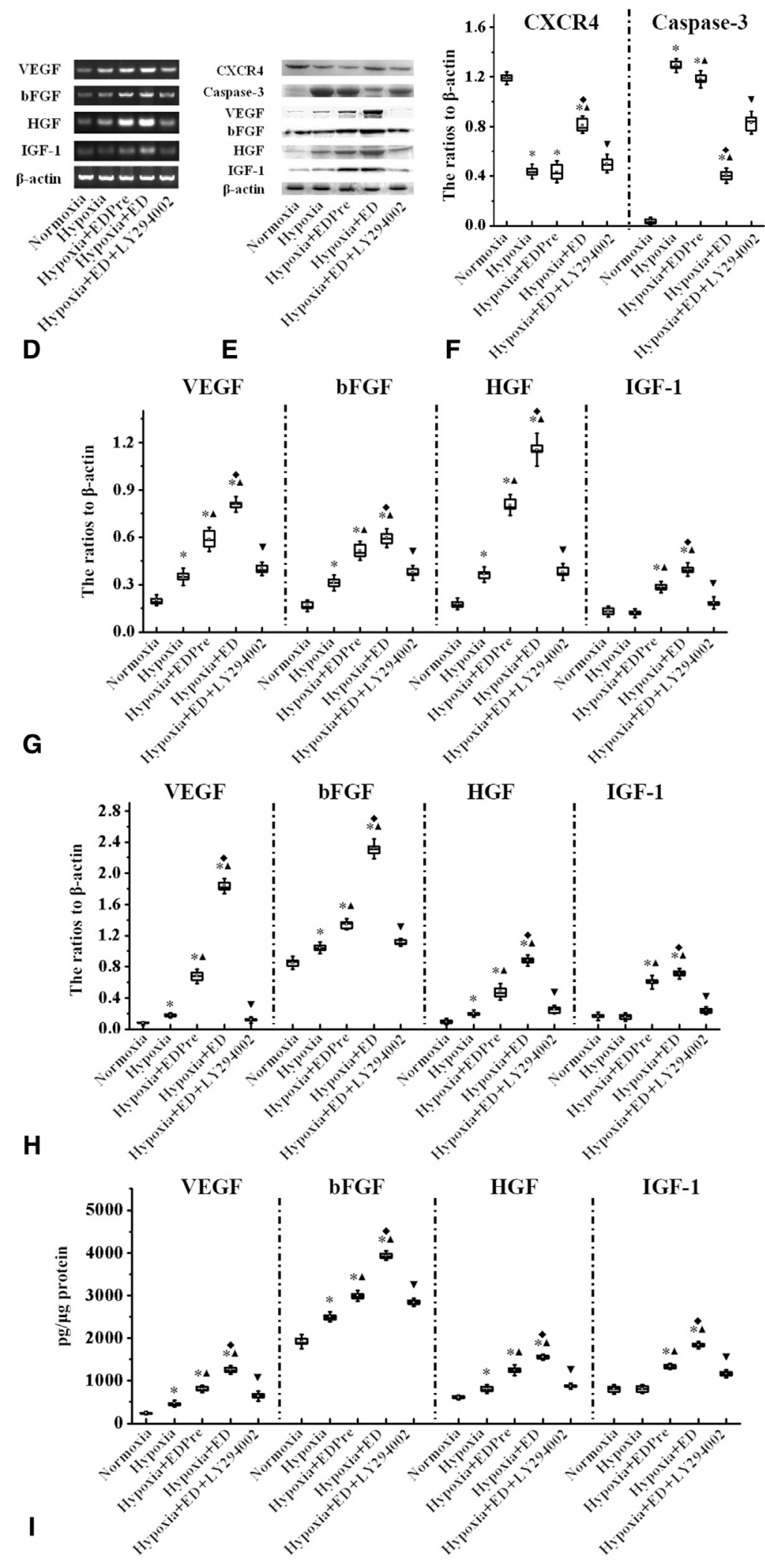

FIGURE 3. (continued). 


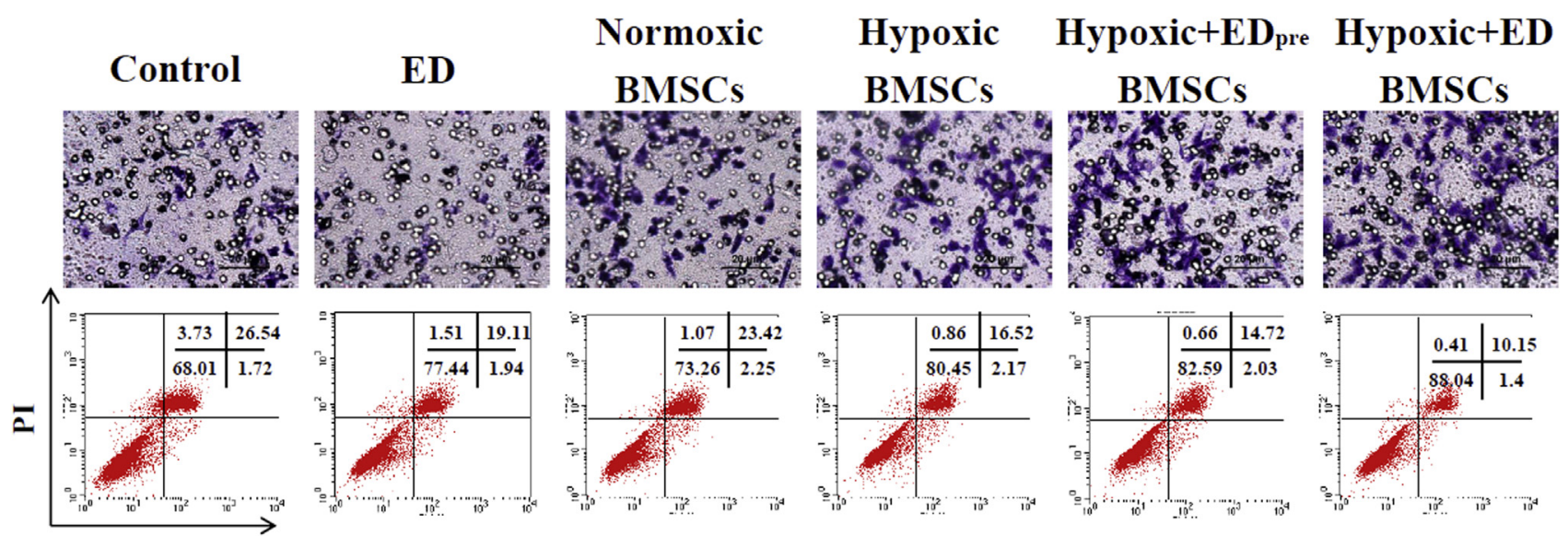

Annexin V-FITC

A
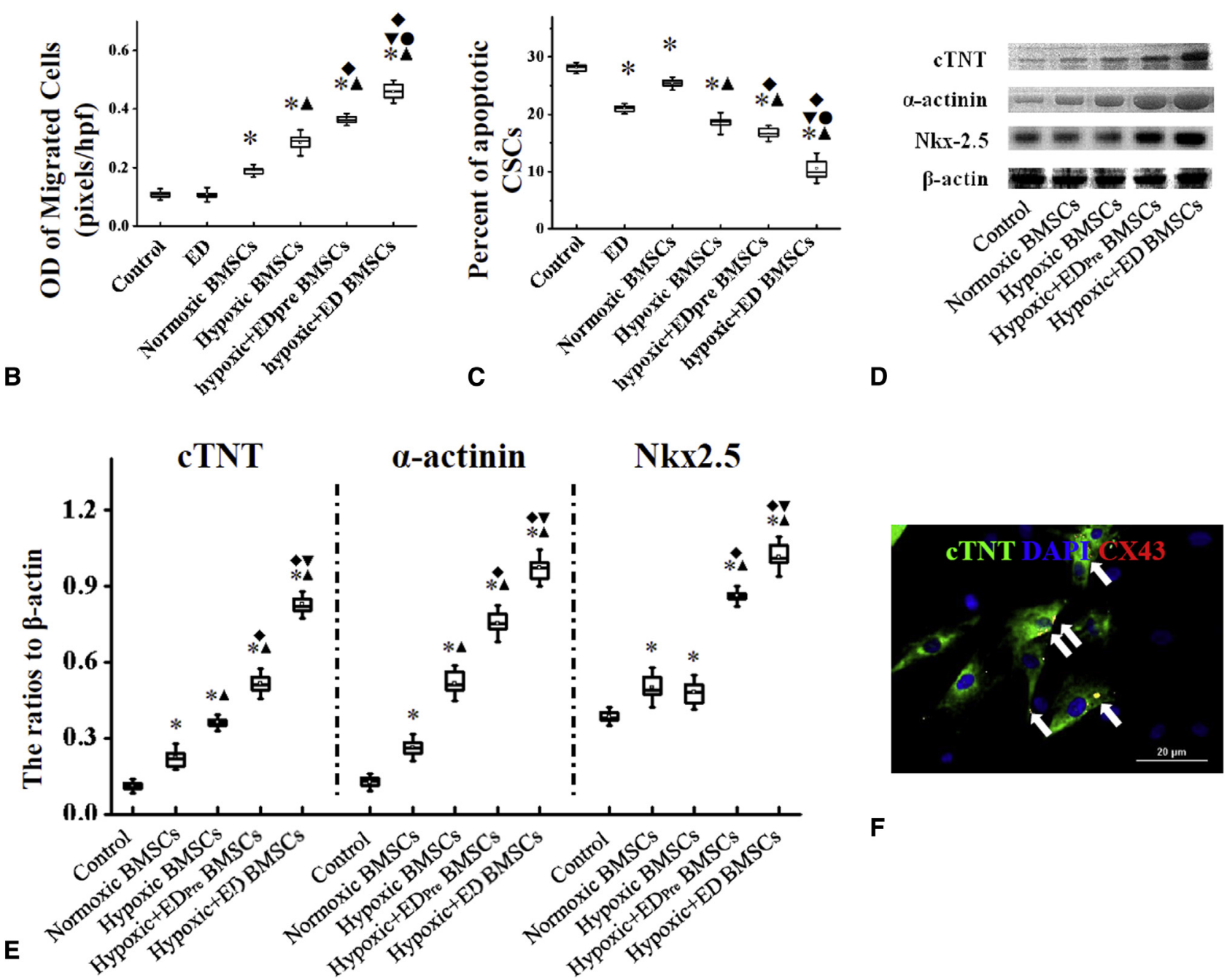

$\mathbf{F}$

FIGURE 4. Ex vivo evaluation of CSC activation induced by BMSCs. Representative graphs of CSC migration (top) and apoptosis (below) exhibited in (A), and quantitative analyses of both were performed in B and C, respectively. Representative photographs of Western blot are shown in (D). E, ratios of cTNT, $\alpha$-actinin, and Nkx2.5 to $\beta$-Actin. F, anti-cTNT (green) and anti-CX43 (red) immunofluorescence staining of CSCs. EDpre, Edaravone pretreatment; $B M S C$, bone marrow mesenchymal stem cell; $O D$, optical density; $C S C$, cardiac stem cells; $c T N T$, cardiac troponin T. $* P<.001$ versus control. ${ }^{\star} P<.001$ versus normoxic BMSCs. ${ }^{\bullet} P$ <.001 versus hypoxic BMSCs. ${ }^{\nabla}<.001$ versus hypoxic + pretreatment edaravone BMSCs. ${ }^{\bullet} P<.001$ versus edaravone. 


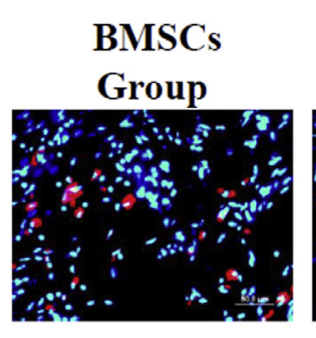

A
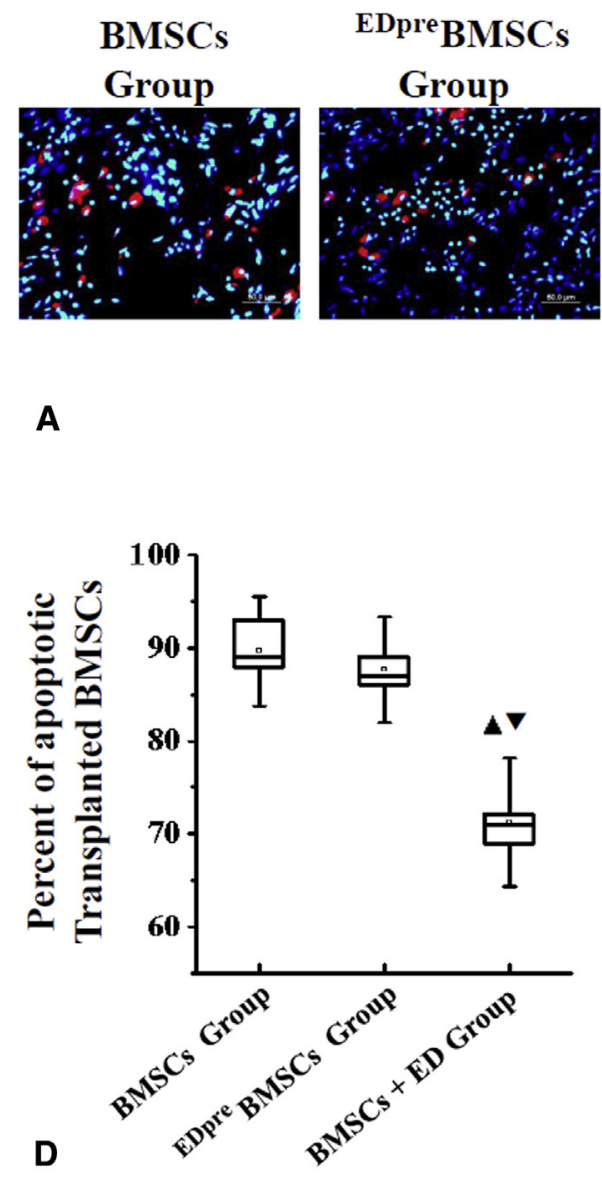
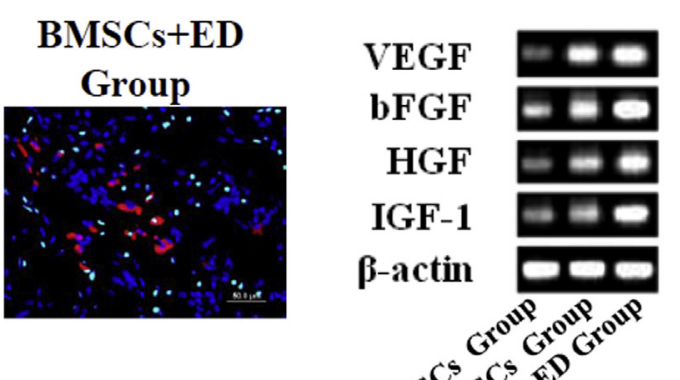

B
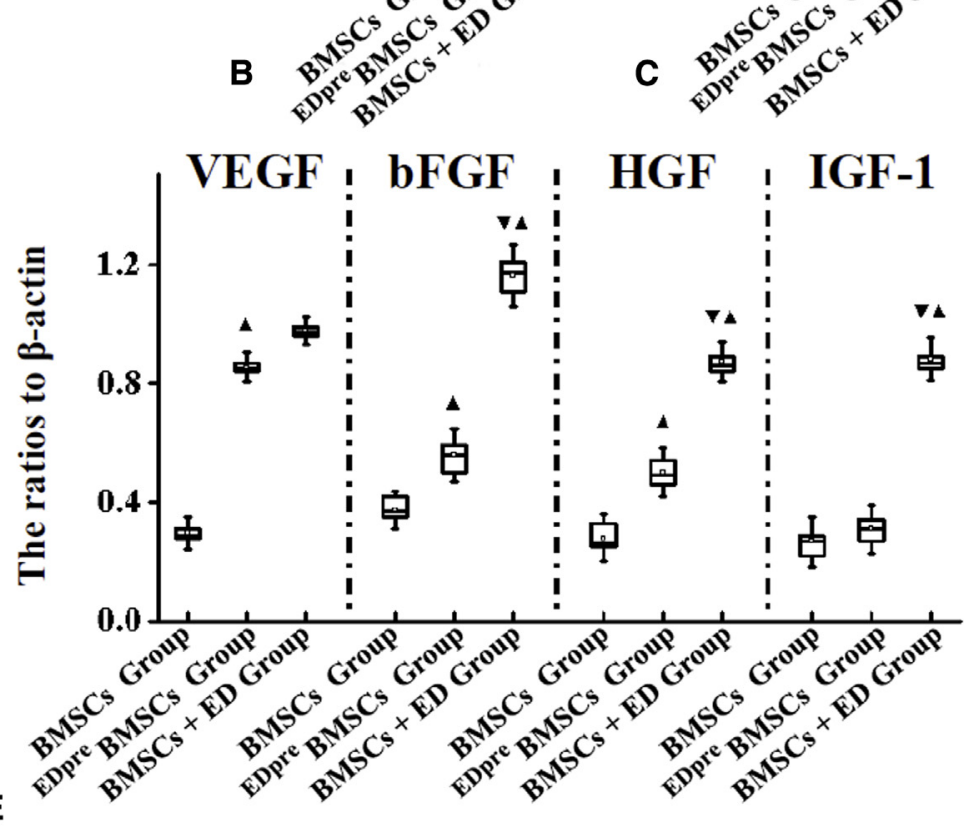

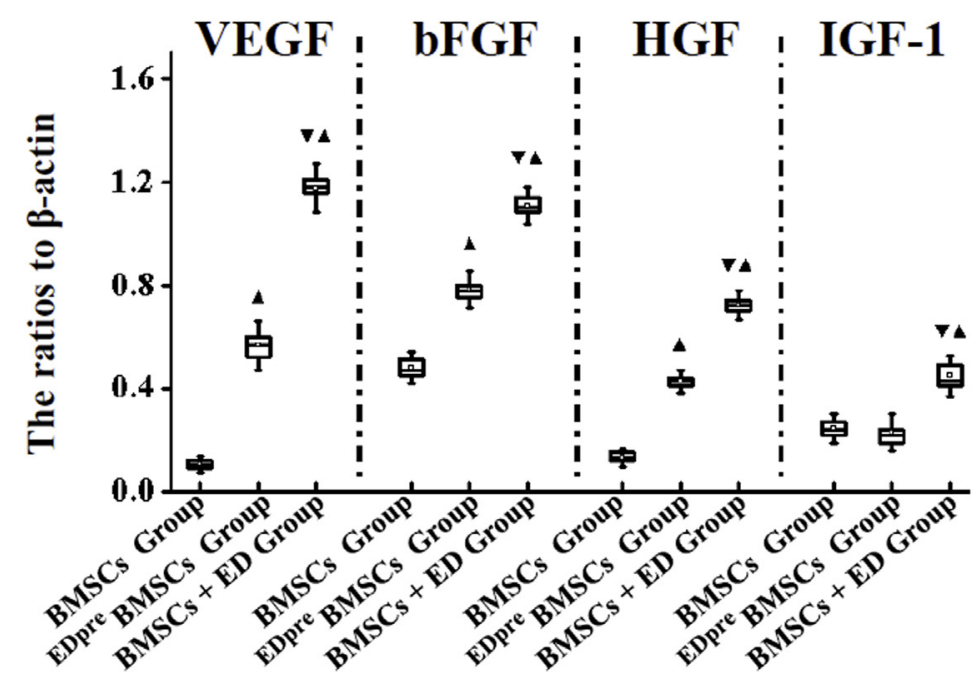

FIGURE 5. In vivo determination of BMSC apoptosis and paracrine function 24 hours after transplantation. Representative photographs of TUNEL staining $(\times 100)$ for the BMSC group, pretreatment edaravone BMSC group, and BMSC + edaravone group are shown in (A). Results of real-time polymerase chain reaction and Western blotting for the 3 groups are shown in $(\mathrm{B})$ and $(\mathrm{C})$, respectively. The ratios for messenger RNA and protein products are compared in (E) and (F), respectively. BMSC, Bone marrow mesenchymal stem cell; $E D p r e$, edaravone pretreatment; $V E G F$, vascular endothelial growth factor; $b F G F$, basic fibroblast growth factor; $H G F$, hepatic growth factor; $I G F-1$, insulin-like growth factor-1. $* P<.001$ versus control group. ${ }^{\boldsymbol{\Lambda}} P<.001$ versus BMSC group. ${ }^{\bullet} P$.001 versus edaravone group. ${ }^{\nabla} P<.001$ versus pretreatment edaravone BMSC group. 

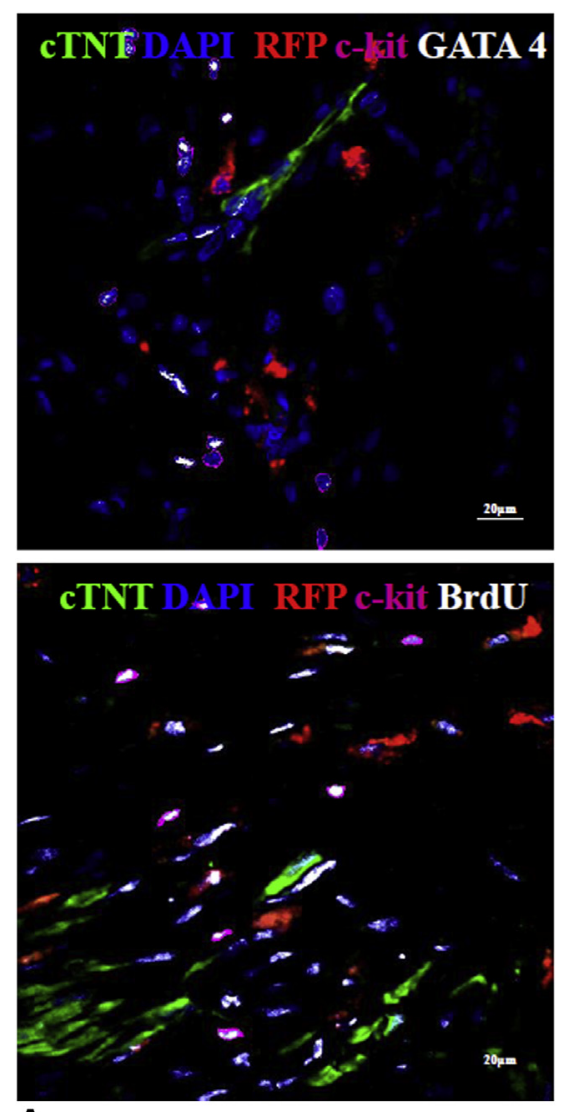

A

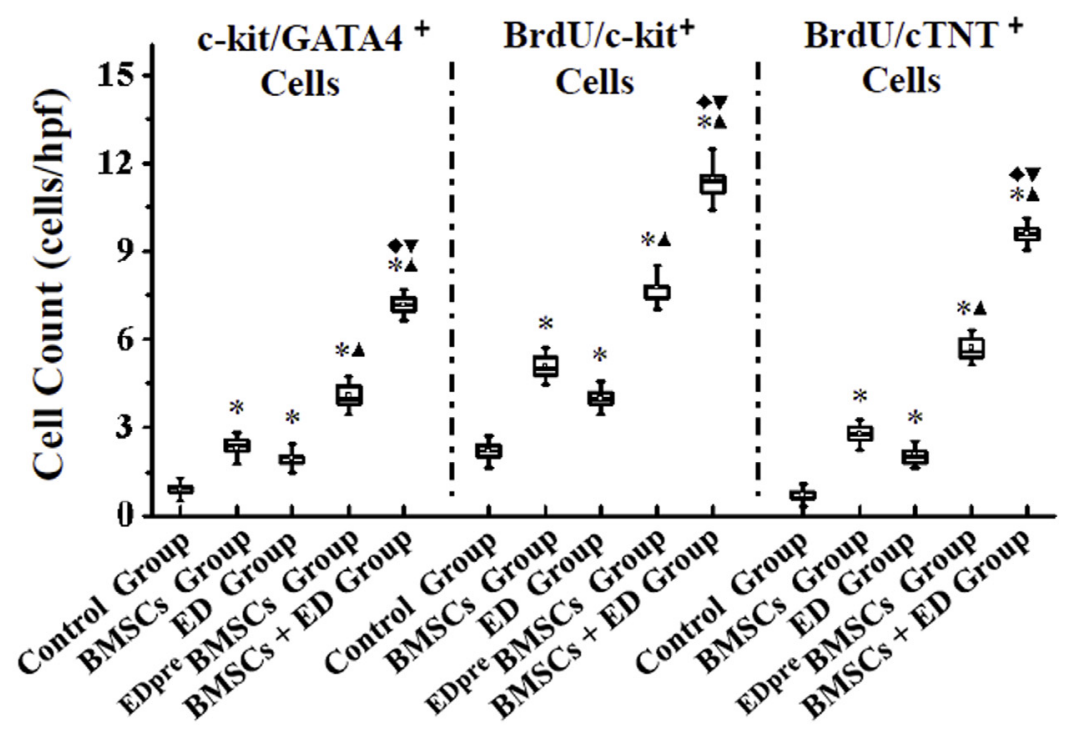

B

FIGURE 6. In vivo analysis of resident CSC-mediated myocardial regeneration 4 weeks after the treatment. A, Representative photographs $(\times 600)$ of immunofluorescence staining of frozen myocardial sections with anti-cTNT (green fluorescence), DAPI (blue fluorescence), RFP (red fluorescence), anti-c-kit (violet fluorescence), and anti-GATA4 or anti-BrdU (white fluorescence). $\mathrm{RFP}^{-}$cells were confirmed to be resident cells, of which c-kit/GATA4 ${ }^{+}$, $\mathrm{BrdU} / \mathrm{c}-\mathrm{kit}^{+}$and BrdU/cTNT ${ }^{+}$cells are the control; BMSCs, ED, pretreatment edaravone BMSC and BMSCs + edaravone groups are compared in (B). $c T N T$, Cardiac troponin T; RFP, red fluorescent protein; $B r d U$, 5-bromo-2-deoxyuridine; BMSC, bone marrow mesenchymal stem cell; EDpre, edaravone pretreatment. ${ }^{\wedge} P<.001$ versus BMSC group. ${ }^{\nabla} P<.001$ versus the pretreatment edaravone BMSC group.

edaravone group, and the edaravone pretreatment BMSC group, compared with the control group $(44.1 \% \pm 2.0 \%$, $P<.001)$. A significant increase was detected in the BMSCs + edaravone group, compared with the other groups $(P<.001$; Figure $7, D)$.

\section{DISCUSSION}

The present study has demonstrated for the first time that edaravone can promote the paracrine potential of the BMSCs via the Akt pathway under an in vitro deep hypoxia/serum deprivation condition when transplanted into an ischemic region, and consequently activate CSCs. Therefore, combined therapy with control-released edaravone and BMSC transplantation may enhance angiogenesis and myocardial regeneration mediated by the resident CSCs and improve left ventricle function, at least during the subacute period.

As a promising therapeutic strategy for acute myocardial infarction, BMSC transplantation has been hampered by apoptosis induced by ischemia and hypoxia within the ischemic regions, owing to incomplete revascularization even after a coronary artery bypass graft operation in patients who suffer from severe and complicated coronary artery disease. Oxidative stress is regarded as a major contributor in hypoxia-induced irreversible damage of the transplanted cells. $^{19}$ Therefore, scavenging excessive intracellular ROS production may provide a protection for the BMSCs against hypoxia in the transplanted areas. Edaravone has been demonstrated to significantly promote protective effects of BMSCs on nerve repair after ischemic injury, via alleviating the hypoxia-induced apoptosis. ${ }^{10,20} \mathrm{In}$ the present study, we found that edaravone could enhance the myocardial repair potential of the transplanted BMSCs as well, resulting in improvement of cardiac function.

As shown by our ex vivo experiment, sustained exposure to edaravone could provide much more protective benefit for the BMSCs against hypoxia-induced injury, compared 


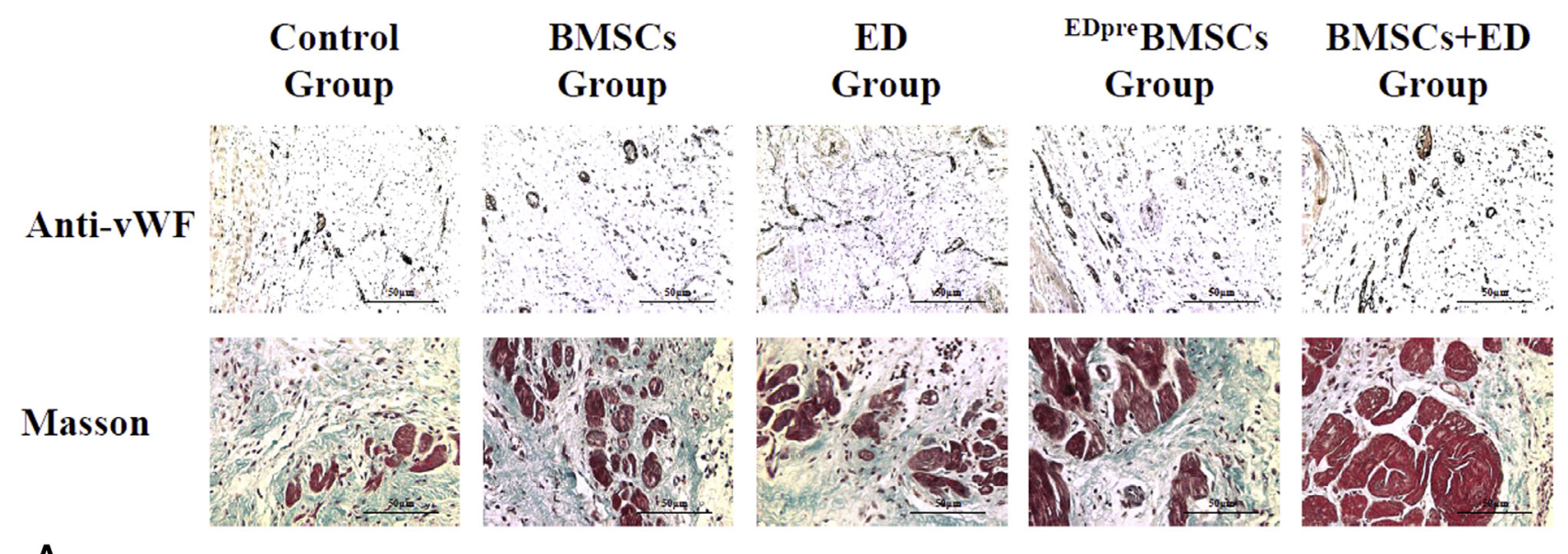

A
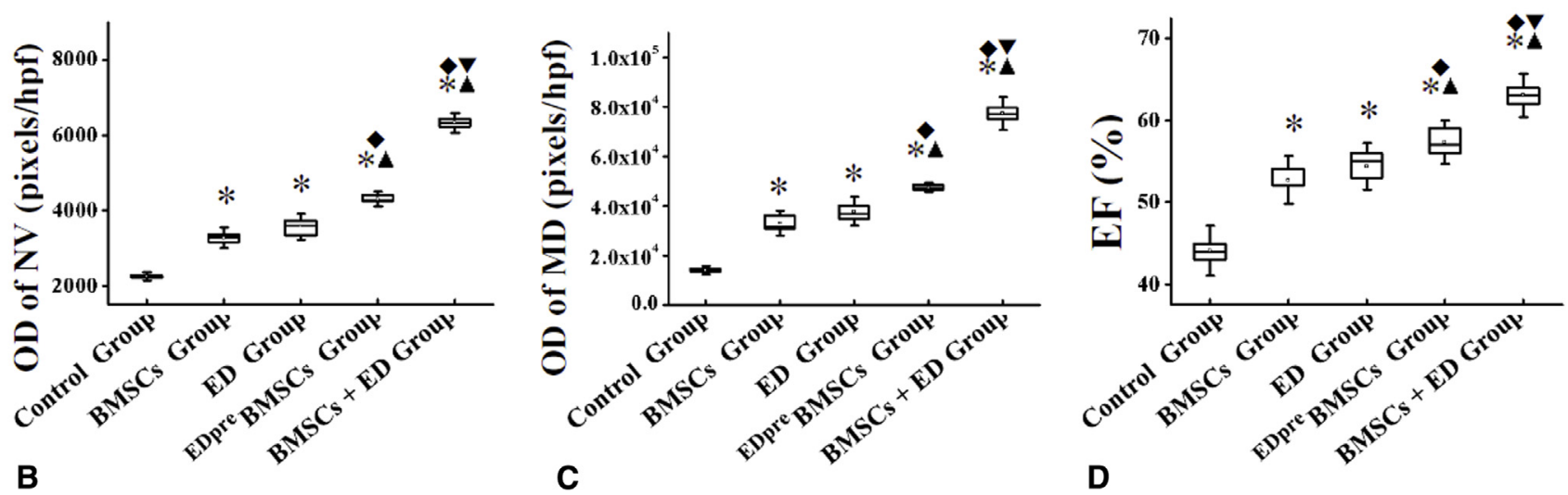

FIGURE 7. In vivo assessment of angiogenesis, myocardium density, and cardiac function. Representative photographs of anti-von Willebrand factor staining (upper row) and Masson trichrome-staining (bottom row: red indicates viable myocardium; blue indicates collagen) of paraffin sections of the ischemic region from the 5 groups are shown in (A) $(\times 200)$. In fact, a few cardiomyocytes were seen in some images, although none were detected in most images. Therefore, we chose a representative image in which the number of cardiomyocytes was approximately the average value. The comparisons of NV and MD among the 5 groups are shown in (B) and (C), respectively. D, The comparison of ejection fraction. $v W F$, von Willebrand factor; $B M S C$, bone marrow mesenchymal stem cell; $E D p r e$, edaravone pretreatment; $O D$, optical density; $N V$, new vessels; $M D$, myocardium density; $E F$, ejection function. ${ }^{*} P<.001$ versus control group. ${ }^{\star} P<.001$ versus BMSC group. ${ }^{\star} P<.001$ versus edaravone group. ${ }^{*} P<.001$ versus the edaravone pretreatment BMSC group.

with pretreatment. In vivo experiments have revealed that controlled release of edaravone was far superior to pretreatment, suggesting that the benefits provided by edaravone are time dependent. Simple local injection clearly will result in a rather short half-time, which cannot completely fulfill the biological function of the agents. Instead, sustained release is important for the local administration of edaravone.

Although suppression of apoptosis is generally agreed to play a crucial role in BMSC transplantation, our study strongly suggests that the activation of the BMSC paracrine pathway may be a more important mechanism, based on the fact that the main effect of BMSCs is via paracrine function. ${ }^{21}$ BMSCs have been demonstrated to secrete many types of cytokines, including VEGF, b-FGF, HGF, and IGF-I. ${ }^{5,22-24}$ Such cytokines have been verified to not only promote angiogenesis and myocardial perfusion, but also enhance CSC proliferation, migration, and differentiation, inhibit CSC apoptosis induced by hypoxia, and consequently promote CSC-mediated myocardial regeneration (the main source of regenerated cardiomyocytes after acute myocardial infarction) and improve left ventricle function. ${ }^{12,21,25,26}$ Our ex vivo results have shown that the paracrine function of the BMSCs was activated by deep hypoxia/serum deprivation before the hypoxic injury, and that edaravone could prolong the activated time to further enhance the activation of BMSCs. Such findings have been confirmed by in vivo experiment, strongly suggesting that ROS mediates hypoxia-induced activation of BMSC paracrine function.

The following mechanism may account for the regulated effects of ROS as a second messenger to promote Akt 
phosphorylation. ${ }^{27}$ In the early phase of deep hypoxia/ serum deprivation, before the oxidative damage caused by a high concentration of ROS, a moderate increase in ROS may activate the PI3K/Akt pathway, and subsequently influence gene expression as well as posttranslational modification of proteins. ${ }^{28}$ The PI3K/Akt is a pivotal signaling pathway in various physiologic processes, including cell survival, apoptosis, migration, and endocrine activities, ${ }^{22,23,29}$ and it is involved in the regulation of cell injury induced by hypoxia. ${ }^{30}$

With prolonged hypoxia, however, continuously accumulated ROS becomes excessive and cytotoxic, leading to inhibition of the PI3K/Akt pathway, ${ }^{30}$ decreased cellular viability and induction of apoptosis. ${ }^{31}$ Therefore, alteration of the PI3K/Akt pathway induced by hypoxia is probably a dynamic process, with an early phase of up-regulation and then down-regulated expression of pAkt. For its capacity to scavenge excessive ROS within the BMSCs, edaravone may prolong the early phase of hypoxia and activation of the Akt pathway, ${ }^{32}$ and consequently attenuate BMSC apoptosis, and promote their migration and paracrine function via this pathway. Other pathways may be involved in these processes ${ }^{6,33-35}$ and need to be delineated.

Despite the encouraging results, questions remain. The function of regenerated cardiomyocytes has not been delineated in detail, despite the assessment of the global function of the left ventricle. Evidence has been acquired that edaravone treatment can convert BMSCs to a neuronal phenotype, which was not involved in the present study. Although many other types of cell are involved in paracrine effects of transplanted BMSCs, this study explored only resident CSC activation, so other mechanisms should be researched in the following experiments. The samples from noninjected sites should be observed further, and the effects of various doses of edaravone were not observed in this study; thus, the optimum dose needs to be determined. Finally, the experiment was performed in animal models; differences between human and rat hearts has not been taken into account.

Edaravone can delay activation of the Akt pathway and cell damage induced by hypoxia, to prolong and enhance activation of BMSCs to further promote angiogenesis and CSC-mediated myocardial regeneration. The combination of controlled release of edaravone and BMSC transplantation may improve left ventricle function significantly after acute myocardial infarction, and provide a new strategy for myocardial regeneration.

\section{Conflict of Interest Statement}

Authors have nothing to disclose with regard to commercial support.
The technical assistance of Xue-Yuan Li, Yong-Hua Bi, Xiao-Yu Guan, Lei Yu, Da-Qing Jiang, and Feng Lv is gratefully acknowledged.

\section{References}

1. Donndorf P, Kundt G, Kaminski A, Yerebakan C, Liebold A, Steinhoff G, et al. Intramyocardial bone marrow stem cell transplantation during coronary artery bypass surgery: a meta-analysis. J Thorac Cardiovasc Surg. 2011;142:911-20.

2. Heldman AW, DiFede DL, Fishman JE, Zambrano JP, Trachtenberg BH, Karantalis V, et al. Transendocardial mesenchymal stem cells and mononuclear bone marrow cells for ischemic cardiomyopathy: the TAC-HFT randomized trial. JAMA. 2014;311:62-73.

3. Chen J, Baydoun AR, Xu R, Deng L, Liu X, Zhu W, et al. Lysophosphatidic acid protects mesenchymal stem cells against hypoxia and serum deprivation-induced apoptosis. Stem Cells. 2008;26:135-45.

4. Toma C, Pittenger MF, Cahill KS, Byrne BJ, Kessler PD. Human mesenchymal stem cells differentiate to a cardiomyocyte phenotype in the adult murine heart. Circulation. 2002;105:93-8.

5. Hu X, Yu SP, Fraser JL, Lu Z, Ogle ME, Wang JA, et al. Transplantation of hypoxia-preconditioned mesenchymal stem cells improves infarcted heart function via enhanced survival of implanted cells and angiogenesis. J Thorac Cardiovasc Surg. 2008;135:799-808.

6. Takahashi G, Sakurai M, Abe K, Itoyama Y, Tabayashi K. MCI-186 prevents spinal cord damage and affects enzyme levels of nitric oxide synthase and $\mathrm{Cu} / \mathrm{Zn}$ superoxide dismutase after transient ischemia in rabbits. J Thorac Cardiovasc Surg. 2003;126:1461-6.

7. Suzuki T, Kazui T, Yamamoto S, Washiyama N, Ohkura K, Ohishi K, et al. Effect of prophylactically administered edaravone during antegrade cerebral perfusion in a canine model of old cerebral infarction. J Thorac Cardiovasc Surg. 2007; 133:710-6.

8. Yano M, Okuda S, Oda T, Tokuhisa T, Tateishi H, Mochizuki M, et al. Correction of defective interdomain interaction within ryanodine receptor by antioxidant is a new therapeutic strategy against heart failure. Circulation. 2005; 112:3633-43.

9. Kotani Y, Ishino K, Osaki S, Honjo O, Suezawa T, Kanki K, et al. Efficacy of MCI-186, a free-radical scavenger and antioxidant, for resuscitation of nonbeating donor hearts. J Thorac Cardiovasc Surg. 2007;133:1626-32.

10. Shen LH, Ye M, Ding XS, Han Q, Zhang C, Liu XF, et al. Protective effects of MCI-186 on transplantation of bone marrow stromal cells in rat ischemic stroke model. Neuroscience. 2012;223:315-24.

11. Sun X, Fang B, Zhao X, Zhang G, Ma H. Preconditioning of mesenchymal stem cells by sevoflurane to improve their therapeutic potential. PLoS One. 2014;9: e90667.

12. Ellison GM, Torella D, Dellegrottaglie S, Perez-Martinez C, Perez de Prado A, Vicinanza C, et al. Endogenous cardiac stem cell activation by insulin-like growth factor- $1 /$ hepatocyte growth factor intracoronary injection fosters survival and regeneration of the infarcted pig heart. J Am Coll Cardiol. 2011;58:977-86.

13. Pouly J, Bruneval P, Mandet C, Proksch S, Peyrard S, Amrein C, et al. Cardiac stem cells in the real world. J Thorac Cardiovasc Surg. 2008;135:673-8.

14. Qi X, Okamoto Y, Murakawa T, Wang F, Oyama O, Ohkawa R, et al. Sustained delivery of sphingosine-1-phosphate using poly(lactic-co-glycolic acid)-based microparticles stimulates Akt/ERK-eNOS-mediated angiogenesis and vascular maturation restoring blood flow in ischemic limbs of mice. Eur J Pharmacol. 2010;634:121-31.

15. Zhang GW, Liu XC, Li-Ling J, Luan Y, Ying YN, Wu XS, et al. Mechanisms of the protective effects of BMSCs promoted by TMDR with heparinized bFGF-incorporated stent in pig model of acute myocardial ischemia. J Cell Mol Med. 2011;15:1075-86.

16. Zhang W, Su X, Gao Y, Sun B, Yu Y, Wang X, et al. Berberine protects mesenchymal stem cells against hypoxia-induced apoptosis in vitro. Biol Pharm Bull. 2009;32:1335-42.

17. Wei N, Yu SP, Gu X, Taylor TM, Song D, Liu XF, et al. Delayed intranasal delivery of hypoxic-preconditioned bone marrow mesenchymal stem cells enhanced cell homing and therapeutic benefits after ischemic stroke in mice. Cell Transplant. 2013;22:977-91.

18. Sun HY, Wang NP, Halkos M, Kerendi F, Kin H, Guyton RA, et al Postconditioning attenuates cardiomyocyte apoptosis via inhibition of JNK and p38 mitogen-activated protein kinase signaling pathways. Apoptosis. 2006;11: 1583-93. 
19. Kim MH, Kim MO, Heo JS, Kim JS, Han HJ. Acetylcholine inhibits long-term hypoxia-induced apoptosis by suppressing the oxidative stress-mediated MAPKs activation as well as regulation of Bcl-2, c-IAPs, and caspase-3 in mouse embryonic stem cells. Apoptosis. 2008;13:295-304.

20. Tian F, Yamashita T, Deguchi K, Omote Y, Kawai H, Ohta Y, et al. In vivo optical imaging correlates with improvement of cerebral ischemia treated by intravenous bone marrow stromal cells (BMSCs) and edaravone. Neurol Res. 2013;35: 1051-8.

21. Hatzistergos KE, Quevedo H, Oskouei BN, Hu Q, Feigenbaum GS, Margitich IS, et al. Bone marrow mesenchymal stem cells stimulate cardiac stem cell proliferation and differentiation. Circ Res. 2010;107:913-22.

22. Kinnaird T, Stabile E, Burnett MS, Lee CW, Barr S, Fuchs S, et al. Marrow-derived stromal cells express genes encoding a broad spectrum of arteriogenic cytokines and promote in vitro and in vivo arteriogenesis through paracrine mechanisms. Circ Res. 2004;94:678-85.

23. Gnecchi M, He H, Noiseux N, Liang OD, Zhang L, Morello F, et al. Evidence supporting paracrine hypothesis for Akt-modified mesenchymal stem cell-mediated cardiac protection and functional improvement. FASEB. 2006; 20:661-9.

24. Wang M, Tan J, Coffey A, Fehrenbacher J, Weil BR, Meldrum DR. Signal transducer and activator of transcription 3-stimulated hypoxia inducible factor-1alpha mediates estrogen receptor-alpha-induced mesenchymal stem cell vascular endothelial growth factor production. J Thorac Cardiovasc Surg. 2009; 138:163-71.e1.

25. Zhang GW, Wen T, Gu TX, Li-Ling J, Wang C, Zhao Y, et al. Transmyocardial drilling revascularization combined with heparinized bFGF-incorporating stent activates resident cardiac stem cells via SDF-1/CXCR4 axis. Exp Cell Res. 2012;318:391-9.

26. Tarui S, Ishigami S, Ousaka D, Kasahara S, Ohtsuki S, Sano S, et al. Transcoronary infusion of cardiac progenitor cells in hypoplastic left heart syndrome: three-year follow-up of the Transcoronary Infusion of Cardiac Progenitor Cells in Patients With Single-Ventricle Physiology (TICAP) trial. J Thorac Cardiovasc Surg. 2015;150:1198-207.
27. Iiyama M, Kakihana K, Kurosu T, Miura O. Reactive oxygen species generated by hematopoietic cytokines play roles in activation of receptor-mediated signaling and in cell cycle progression. Cell Signal. 2006;18:174-82.

28. Sies H. Oxidative stress: oxidants and antioxidants. Exp Physiol. 1997;82:291-5.

29. Mares JM, Foley LS, Bell MT, Bennett DT, Freeman KA, Meng X, et al. Erythropoietin activates the phosporylated cAMP [adenosine $3^{\prime} 5^{\prime}$ cyclic monophosphate] response element-binding protein pathway and attenuates delayed paraplegia after ischemia-reperfusion injury. J Thorac Cardiovasc Surg. 2015;149:920-4.

30. Hou M, Cui J, Liu J, Liu F, Jiang R, Liu K, et al. Angiopoietin-like 4 confers resistance to hypoxia/serum deprivation-induced apoptosis through PI3K/Akt and ERK1/2 signaling pathways in mesenchymal stem cells. PLoS One. 2014; 9:e85808.

31. Droge W. Free radicals in the physiological control of cell function. Physiol Rev. 2002;82:47-95

32. Niyaz M, Numakawa T, Matsuki Y, Kumamaru E, Adachi N, Kitazawa H, et al. MCI-186 prevents brain tissue from neuronal damage in cerebral infarction through the activation of intracellular signaling. J Neurosci Res. 2007;85: 2933-42.

33. Liu XY, Yao LL, Chen YJ, Tao BB, Yu YC, Bian WH, et al. Survivin is involved in the anti-apoptotic effect of edaravone in PC12 cells. Mol Cell Biochem. 2009; 327:21-8.

34. Wen J, Watanabe K, Ma M, Yamaguchi K, Tachikawa H, Kodama M, et al. Edaravone inhibits JNK-c-Jun pathway and restores anti-oxidative defense after ischemia-reperfusion injury in aged rats. Biol Pharm Bull. 2006;29:713-8.

35. Cheng SE, Lee IT, Lin CC, Wu WL, Hsiao LD, Yang CM. ATP mediates NADPH oxidase/ROS generation and COX-2/PGE2 expression in A549 cells: role of P2 receptor-dependent STAT3 activation. PloS One. 2013;8: e54125.

Key Words: myocardial infarction, stem cell transplantation, edaravone, regeneration, ventricular function 